\title{
Genetic Correlates of Psychological Responses to the COVID-19 Crisis in Young Adult Twins in Great Britain
}

\author{
Kaili Rimfeld ${ }^{1}$ (1) Margherita Malanchini ${ }^{1,2} \cdot$ Andrea G. Allegrini $^{1} \cdot$ Amy E. Packer $^{1} \cdot$ Andrew McMillan $^{1}$. \\ Rachel Ogden ${ }^{1} \cdot$ Louise Webster $^{1}$ - Nicholas G. Shakeshaft ${ }^{1,3} \cdot$ Kerry L. Schofield $^{1,3}$. Jean-Baptiste Pingault ${ }^{4}$. \\ Argyris Stringaris $^{5} \cdot$ Sophie von Stumm ${ }^{6} \cdot$ Robert Plomin $^{1}$
}

Received: 13 October 2020 / Accepted: 1 February 2021 / Published online: 24 February 2021

(c) The Author(s) 2021

\begin{abstract}
We investigated how the COVID-19 crisis and the extraordinary experience of lockdown affected young adults in England and Wales psychologically. One month after lockdown commenced (T2), we assessed 30 psychological and behavioural traits in more than 4000 twins in their mid-twenties and compared their responses to the same traits assessed in 2018 (T1). Mean changes from $\mathrm{T} 1$ to $\mathrm{T} 2$ were modest and inconsistent. Contrary to the hypothesis that major environmental changes related to COVID-19 would result in increased variance in psychological and behavioural traits, we found that the magnitude of individual differences did not change from $\mathrm{T} 1$ to $\mathrm{T} 2$. Twin analyses revealed that while genetic factors accounted for about half of the reliable variance at $\mathrm{T} 1$ and $\mathrm{T} 2$, they only accounted for 15\% of individual differences in change from $\mathrm{T} 1$ to $\mathrm{T} 2$, and that nonshared environmental factors played a major role in psychological and behavioural changes. Shared environmental influences had negligible impact on T1, T2 or T2 change. Genetic factors correlated on average .86 between T1 and $\mathrm{T} 2$ and accounted for over half of the phenotypic stability, as would be expected for a 2-year interval even without the major disruption of lockdown. We conclude that the first month of lockdown has not resulted in major psychological or attitudinal shifts in young adults, nor in major changes in the genetic and environmental origins of these traits. Genetic influences on the modest psychological and behavioural changes are likely to be the result of gene-environment correlation not interaction.
\end{abstract}

Keywords Response to global pandemic · Depression · Life satisfaction · Psychological stress · Psychopathology · COVID-19

\section{Introduction}

It is rare for such massive and abrupt social change to occur as the world has experienced with the COVID-19 pandemic and lockdown. COVID-19 disease can be a life or death

Edited by Yoon-Mi Hur.

Kaili Rimfeld and Margherita Malanchini are joint first authors.

Kaili Rimfeld

kaili.rimfeld@kcl.ac.uk

1 Social, Genetic and Developmental Psychiatry, Institute of Psychiatry, Psychology \& Neuroscience, King's College London, 16 De Crespigny Park, London SE5 8AF, UK

2 School of Biological and Chemical Sciences, Queen Mary University of London, Mile End Road, London E1 4NS, UK

3 Quodit Ltd., 71-74 Shelton Street, Covent Garden, London WC2H 9JQ, UK issue for those infected with the virus, but the psychological responses of those infected and of the many more people in lockdown who have not contracted the disease are also of concern. For example, a recent review of 24 studies on the effects of quarantine concluded that 'the psychological impact of quarantine is wide-ranging, substantial, and can be long lasting' (Brooks et al. 2020). Low mood and irritability stood out with an incidence of $73 \%$ and $57 \%$, respectively,

4 Clinical, Educational \& Health Psychology, Division of Psychology \& Language Sciences, Faculty of Brain Sciences, University College London, 26 Bedford Way, London WC1H ODS, UK

5 Mood, Brain \& Development Unit, Emotion and Development Branch, National Institute of Mental Health, 9000 Rockville Pike, Building 15K, Bethesda, MD 20892, USA

6 Psychology in Education Research Centre, Department of Education, University of York, York YO10 5DD, UK 
but negative effects were also found for diverse measures including stress, anxiety and insomnia, with some indication of long-term effects such as post-traumatic stress and drug abuse. The Office for National Statistics reports that the cases of depression almost doubled, just two months after lockdown (Office for National Statistics 2020). Also, many studies have found increased post-traumatic stress symptoms following natural disasters such as earthquakes and manmade disasters such as terrorism (Furr et al. 2010). These events can also affect several aspects of mental health as well as substance abuse both in the short and long term (Neria et al. 2009).

Research suggests that the COVID-19 pandemic will worsen psychological health on average in a population (Holmes et al. 2020; Galea et al. 2020). Social and physical distancing have abruptly interrupted normal lives and social opportunities essential for normal psychological functioning. However, the crisis is likely to affect individuals differently (Holmes et al. 2020). Individual differences are likely to be large, possibly even including some people whose psychological health is improved by the crisis (e.g. increased physical activity, volunteering, community satisfaction). An important issue is that the causes of mean differences can be unrelated to the causes of individual differences. For example, the cause of mean changes before and after the COVID-19 crisis can safely be attributed to the environmental effects of the pandemic and lockdown. However, this does not imply that differences in pandemic experiences are the sole source of individual differences in response to the crisis. Importantly, the way in which individuals react to the same event can depend on their genetics.

Here we investigated genetic as well as environmental influences on individual differences in psychological and behavioural traits before the COVID-19 crisis and lockdown (T1) and one month after lockdown had commenced in the UK (T2). In addition to asking participants how they think the crisis affected them, we compared the same psychological and behavioural traits obtained at $\mathrm{T} 1$ and $\mathrm{T} 2$ on the same individuals with data at both $\mathrm{T} 1$ and $\mathrm{T} 2$. To assess the aetiology of individual differences, we used the classical twin design based on the resemblance of identical and non-identical twins. From 17 April to 4 May 2020, we collected online data from 4000 twins in our Twins Early Development Study (TEDS; Rimfeld et al. 2019) from whom we already had data at $\mathrm{T} 1$. We included 30 diverse psychological constructs, such as anxiety, depression, well-being, alcohol use (frequency and quantity), relationships, achievement motivation, purpose in life, life goals, physical activity, online behaviour, volunteering, and community satisfaction. These same measures had been included in a 2018 wave of assessment in TEDS (T1).

The twins were born between 1994 and 1996. They were thus in their early twenties during $\mathrm{T} 1$ and $\mathrm{T} 2$. Few twin studies have focused on this age when the twins are completing their studies and beginning their adult life, entering the workforce, and forming long-term relationships. At this tipping point in their lives, it could be argued, they have the most to lose from the crisis personally, socially and economically.

We describe mean changes from $\mathrm{T} 1$ to $\mathrm{T} 2$, hypothesising that changes will be modest and inconsistent, with some positive as well as negative changes (Hypothesis 1). However, our focus is on individual differences and their genetic and environmental origins at $\mathrm{T} 1$ and $\mathrm{T} 2$ and in changes from $\mathrm{T} 1$ to T2. On the assumption that the COVID-19 crisis affected people differently, we hypothesised that variance will be greater at $\mathrm{T} 2$ than $\mathrm{T} 1$ (Hypothesis 2). We predicted that phenotypic correlations will be substantial between $\mathrm{T} 1$ and T2 (Hypothesis 3), indicating the stability of psychological traits across the 2-year period.

Our overall hypothesis is that genetics, by which we mean inherited DNA differences, is the major systematic force governing how people respond psychologically to the COVID-19 crisis. Specifically, we expected that all traits will show substantial genetic influence at $\mathrm{T} 1$, as indicated by a large body of genetic research on psychological traits (Knopik et al. 2017; Polderman et al. 2015). We also hypothesised that, despite the crisis, genetics will be similarly influential at T2 (Hypothesis 4). We also predicted that heritability will be lower for $\mathrm{T} 1$ to $\mathrm{T} 2$ change scores because they only capture genetic effects at $\mathrm{T} 2$ that are independent of genetic effects at T1. These new genetic effects capture individual differences in response to the pandemic, although they could also be explained by various other environmental effects that happen during this developmental stage or by maturational changes during the 2 years. We operationalised change by regressing $\mathrm{T} 1$ scores from $\mathrm{T} 2$ scores so that $\mathrm{T} 2$ scores are independent of scores at $\mathrm{T} 1$, which we refer to as 'change scores'. Crucially, we predicted that genetic correlations between $\mathrm{T} 1$ and $\mathrm{T} 2$ will be substantial (Hypothesis 5), indicating that, despite the COVID-19 crisis, individual differences at T2 are largely governed by the same genetic factors that affect $\mathrm{T} 1$. That is, we did not expect substantial new genetic factors to explain the same psychological constructs 1 month after the lockdown.

Environmental factors are important too, but we predicted that their effects on individuals are not the systematic effects of family environment. The twin design can be used to distinguish systematic environmental influences attributable to growing up in the same family, called 'shared' environmental influences, from other environmental influences (Plomin and Daniels 1987). Despite a century of the 'nurture assumption' in which the family was assumed to be the major systematic source of environmental influence (Harris 1998), shared environmental influences are generally negligible, and especially as 
young adults leave their family and make their own way in the world (Plomin 2018). This is the rationale for our hypothesis that such shared environmental influences will have negligible impact at both $\mathrm{T} 1$ and $\mathrm{T} 2$ as well as for change from T1 to T2 (Hypothesis 6). Although environmental effects are substantial, our hypothesis is that the environmental effects that make a difference are largely 'non-shared', idiosyncratic and unsystematic (Plomin 2018).

We predicted that similar results will be obtained from bivariate model-fitting analysis (Hypothesis 7). That is, most of the genetic effects on $\mathrm{T} 2$ scores will be accounted for by genetic effects in common with $\mathrm{T} 1$, although there will be some novel genetic effects at $\mathrm{T} 2$ independent of $\mathrm{T} 1$, possibly due to gene-environment interplay. Environmental effects due to shared rearing or living circumstances during lockdown will be negligible.

Finally, we predicted that these results for $\mathrm{T} 2$ change will not interact significantly with potential moderators (Hypothesis 8). Lockdown presents a quasi-experimental test of contemporary shared environments by comparing results for twins living together during lockdown and those living apart. If such shared experiences were important, twins locked down together should be more similar than twins living apart during lockdown. On the basis of the generally weak effects of shared rearing environment, we predicted that environmental effects due to living together during lockdown will be negligible. We also investigated other possible moderators of genetic and environmental influences on individual differences in psychological traits before and during the COVID-19 crisis, such as conditions of lockdown, having COVID-19 symptoms, socioeconomic status and gender. However, our power to detect moderation is limited to large GxE effects (Hanscombe et al. 2012).

All of our hypotheses were preregistered prior to analysis with Open Science Framework: https://osf.io/r58be/.) In summary, they were:

1. Mean changes from $\mathrm{T} 1$ to $\mathrm{T} 2$ will be modest and inconsistent.

2. Variance will be greater at $\mathrm{T} 2$ than $\mathrm{T} 1$.

3. Phenotypic correlations will be substantial between $\mathrm{T} 1$ and $\mathrm{T} 2$.

4. Heritability of individual differences will be substantial for all traits at T1 and T2. Heritability will be lower for $\mathrm{T} 1$ to $\mathrm{T} 2$ change scores.

5. For all traits, genetic correlations between $\mathrm{T} 1$ and $\mathrm{T} 2$ will be high.

6. Environmental influences due to shared rearing or current living circumstances will be negligible for all traits at $\mathrm{T} 1$ and $\mathrm{T} 2$ as well as for $\mathrm{T} 2$ change scores.
7. Similar results will be obtained from a bivariate modelfitting analysis across T1 and T2.

8. These results for $\mathrm{T} 2$ change will not interact significantly with potential moderators.

\section{Results}

\section{Means}

Figure 1 illustrates means and standard deviations for the 30 measures at $\mathrm{T} 1, \mathrm{~T} 2$ and for $\mathrm{T} 2$ change. The details of the descriptive statistics, along with descriptive statistics further broken down by gender and zygosity, are included in Supplementary Tables 1-7. These results are based on one twin randomly selected from each pair so that the data points are independent. Results for the other twin are virtually identical, as shown in Supplementary Tables 8-10.

Almost as many changes were in a positive direction as in a negative direction. However, the effect sizes are modest as indicated by Cohen's $d$ statistic, which is the ratio of the mean difference to the standard deviation (Cohen 1988; Fig. 1). The average $d$ across the 30 measures was 0.24 , which accounts for less than two percent of the variance and includes as many positive as negative changes.

Cohen (1988) proposed, as convention, that a large effect size is a $d$ of 0.8 , accounting for about $25 \%$ of the variance. Only one large negative effect emerged, decreased Volunteering (0.84), which seems likely to be due to less opportunity for volunteering during lockdown.

A $d$ of 0.5 , considered a medium effect size, accounts for about $9 \%$ of the variance. Medium-sized mean differences in the negative direction emerged for three variables. Prosocial Behaviour declined (0.44), which, like Volunteering, might be due in part to reduced opportunity. Achievement Motivation decreased (0.47), which is worrying because emerging adults are our next generation of workers. Verbal Victimisation declined (0.58), which again could be explained by decrease in social interactions, particularly in person, during the lockdown. Hyperactivity-Inattention increased (0.42), which seems to fit with reports that people feel less able to concentrate. Other effect sizes were modest $(d=0.20)$.

\section{Variances}

These mean differences mask a wide range of individual differences. If the COVID-19 crisis affected people in more extreme ways, we would expect to see increased variance at T2. The standard deviations (Supplementary Tables 1 and 2) do not support this hypothesis. The average standard deviation at T2 (1.71) was slightly lower than at T1 (1.79). Out of 30 variables, variance decreased in 17 measures and increased in 13 measures (Supplementary Table 3). Many 


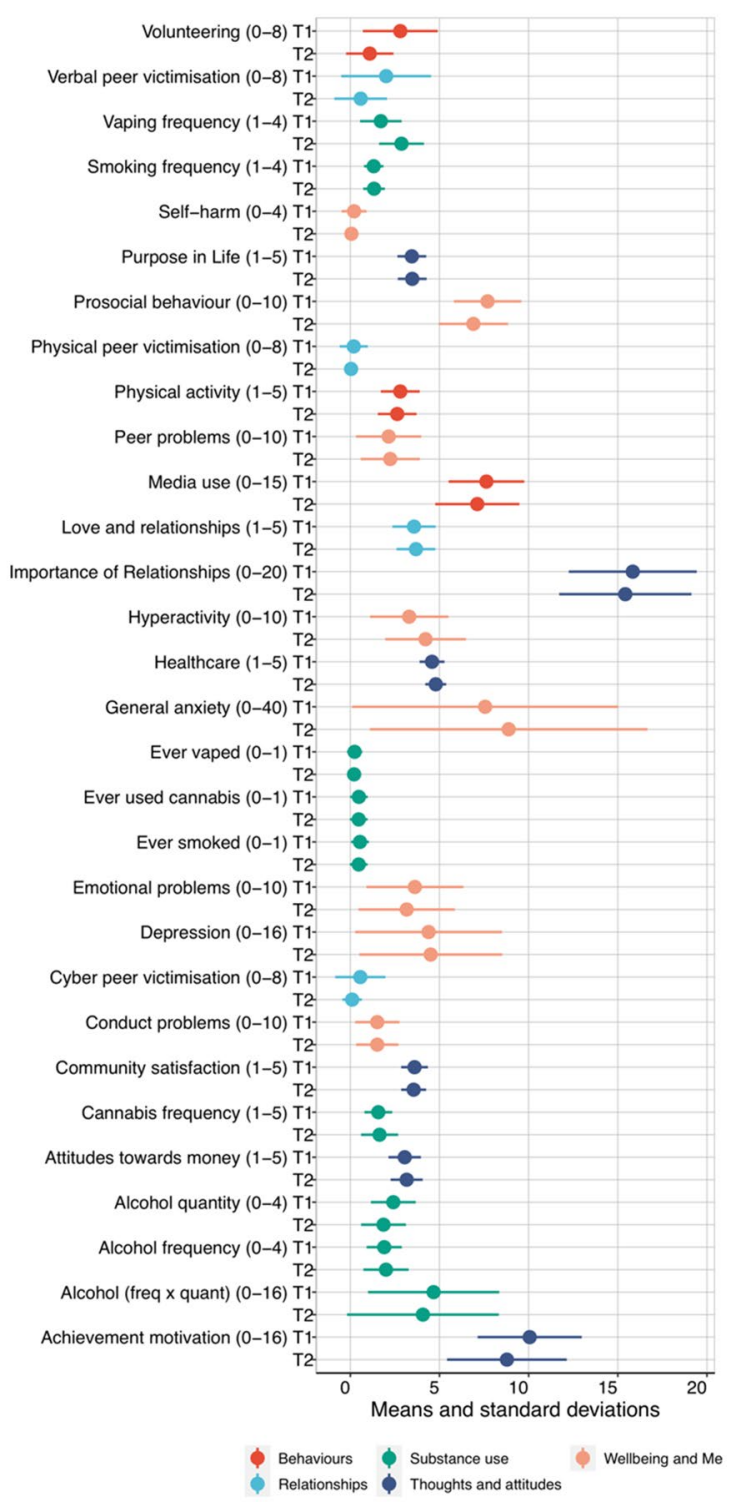

Fig. 1 Descriptive statistics for all measures at T1 and T2 (minimum and maximum scores for each scale in the parentheses). Means and standard deviations for all the measures are presented in the panel on

of these variance differences are significant even after correcting for multiple testing, however, the effect size as indexed by $\mathrm{F}$ value (ratio between variance at $\mathrm{T} 1$ and $\mathrm{T} 2$ ) is small (average $\mathrm{F}$ ratio 1.49 , regardless whether variance increased or decreased from T1 to T2), and the effect sizes were smaller when variance increased from $\mathrm{T} 1$ to $\mathrm{T} 2$ than when variance decreased. These variance differences were similar for males and females (average $\mathrm{F}$ ratio 1.65 for males and 1.52 for females).

For these analyses and the following analyses of individual differences, we focused on variables that showed sufficient variability and approached normal distributions, including Achievement motivation, Alcohol use (measured

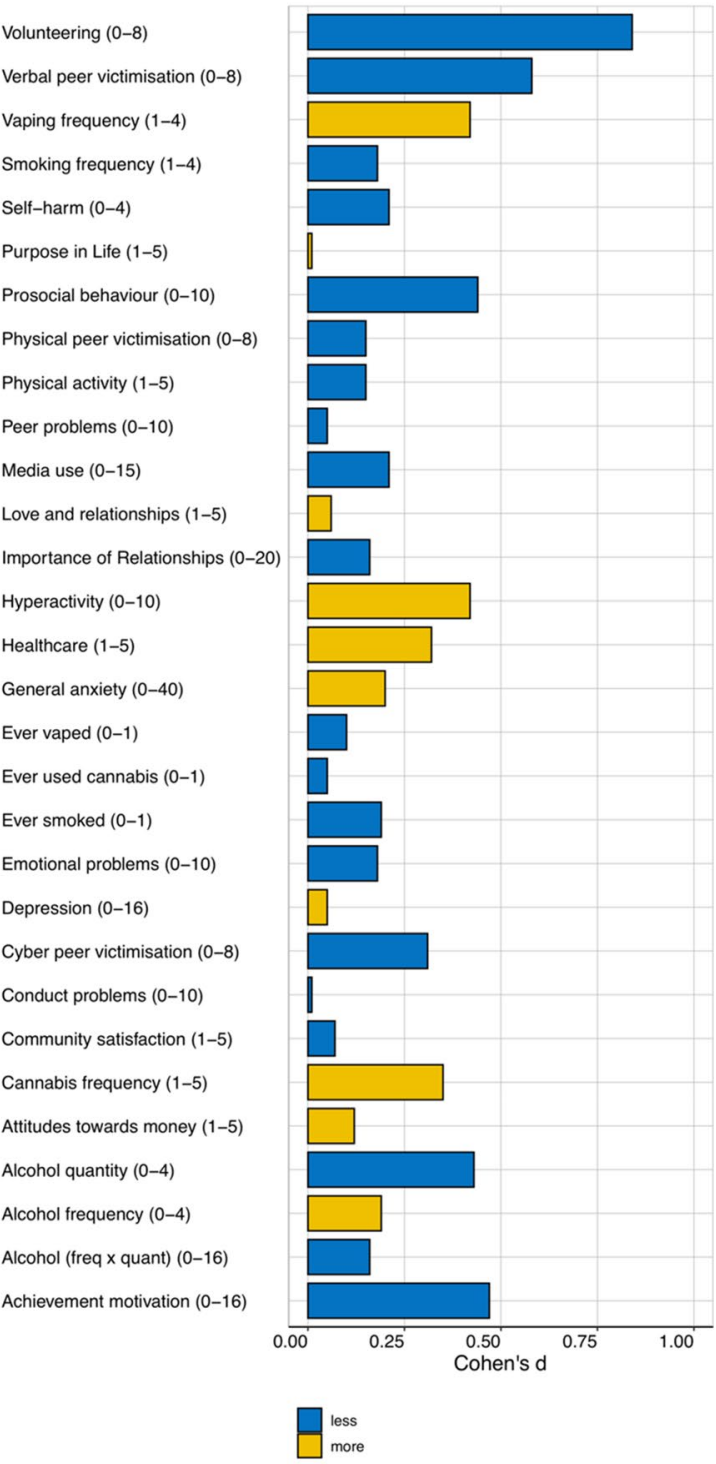

the left. On the right are effect sizes (Cohen's d) for the differences between phenotypes at $\mathrm{T} 1$ and $\mathrm{T} 2$

by multiplying the frequency with quantity), Community satisfaction, Conduct problems, Depression, Emotional problems, General anxiety, Healthcare, Hyperactivity/inattention, Importance of relationships, Love and relationships, Media use, Money attitudes, Peer problems, Physical activity, Prosocial behaviour, Purpose in life and Volunteering.

\section{Covariances}

If the COVID-crisis re-shuffled the rank order of individual differences, we would expect to see little stability from $\mathrm{T} 1$ to $\mathrm{T} 2$. Pearson correlations from $\mathrm{T} 1$ to $\mathrm{T} 2$ are shown in Fig. 2 and listed in Supplementary Table 11, 
Fig. 2 Phenotypic correlations (and 95\% confidence intervals) between measures at $\mathrm{T} 1$ and $\mathrm{T} 2$

\author{
Volunteering \\ Purpose in life \\ Prosocial behaviour \\ Physical activity \\ Peer problems \\ Media use \\ Love and relationships \\ Importance of relationships \\ Hyperactivity \\ Healthcare \\ General anxiety \\ Emotional problems \\ Depression \\ Conduct problems \\ Community satisfaction \\ Attitudes towards money \\ Alcohol freq $x$ quant \\ Achievement motivation
}

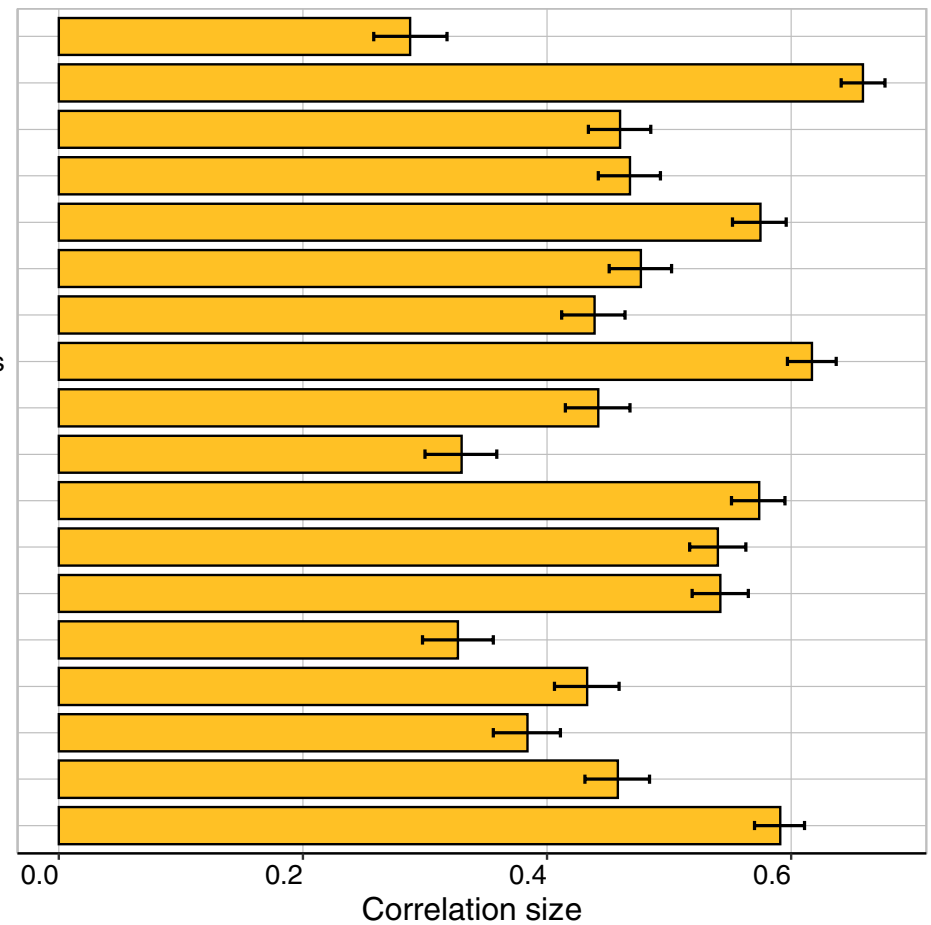

separately for males and females. The average correlation is 0.48 across the 2-year gap. The most stable measures include Purpose in Life (0.68), Emotional Problems (0.56), Peer Problems (0.58), General Anxiety (0.57), and Depression (0.56). Stability correlations were generally similar for males and females, with average stability correlations of 0.50 and 0.47 , respectively.

Reliability of the measures represents a ceiling for stability. In TEDS, we obtained two-week test-retest reliability from TEDS twins on most measures as part of our preparatory work for the 2018 (T1) assessment (Supplementary Table 12). The average test-retest reliability was 0.71 , ranging from 0.47 for Importance of Healthcare to 0.84 for Volunteering. The average stability correlation of 0.48 implies that $48 \%$ of the total variance of the measures was stable from $\mathrm{T} 1$ to $\mathrm{T} 2$. Taking test-retest reliability into account (through dividing the correlation estimate by the test-retest coefficient) suggests that $68 \%$ of the reliable variance of the measures was stable from $\mathrm{T} 1$ to $\mathrm{T} 2$. This finding indicates that there is still some change between $\mathrm{T} 1$ and $\mathrm{T} 2$ across the range of psychological and behavioural measures studied here.

Despite the substantial stability from $\mathrm{T} 1$ to $\mathrm{T} 2$, $\mathrm{T} 2$ change scores revealed some individuals who changed dramatically in positive as well as negative directions, as illustrated in Supplementary Fig. 1.

Although phenotypic moderation of the psychological response to the COVID crisis revealed many significant interactions between moderators and outcome variables, these interactions did not survive correction for multiple testing using Bonferroni corrections. Moreover, the effect sizes of the interaction terms were small, explaining less than $1 \%$ of the variance in all cases (See Supplementary Tables 13-33).

\section{Genetic and environmental aetiologies of variances and covariances}

\section{Twin correlations}

Figure 3 depicts intraclass correlations for identical and non-identical twins at T1 and T2 and for T2 change scores. (See Supplementary Tables 34-36 for the correlation coefficients). We will describe the main results of the twin analysis using these twin correlations, although later we show that these results are confirmed by structural equation modelling, which also provides $95 \%$ confidence intervals for the genetic and environmental estimates.

At T1, the average twin correlations for identical and nonidentical twins were 0.35 and 0.16 , respectively. Because identical twins are identical genetically whereas non-identical twins are only share $50 \%$ of their segregating genes, the difference in their correlations indexes genetic influence on individual differences, called heritability. Doubling the difference between these correlations suggests a rough estimate of heritability of $35 \%$ at $\mathrm{T} 1$ because heritability cannot exceed the identical twin correlation. At T2, the average twin 


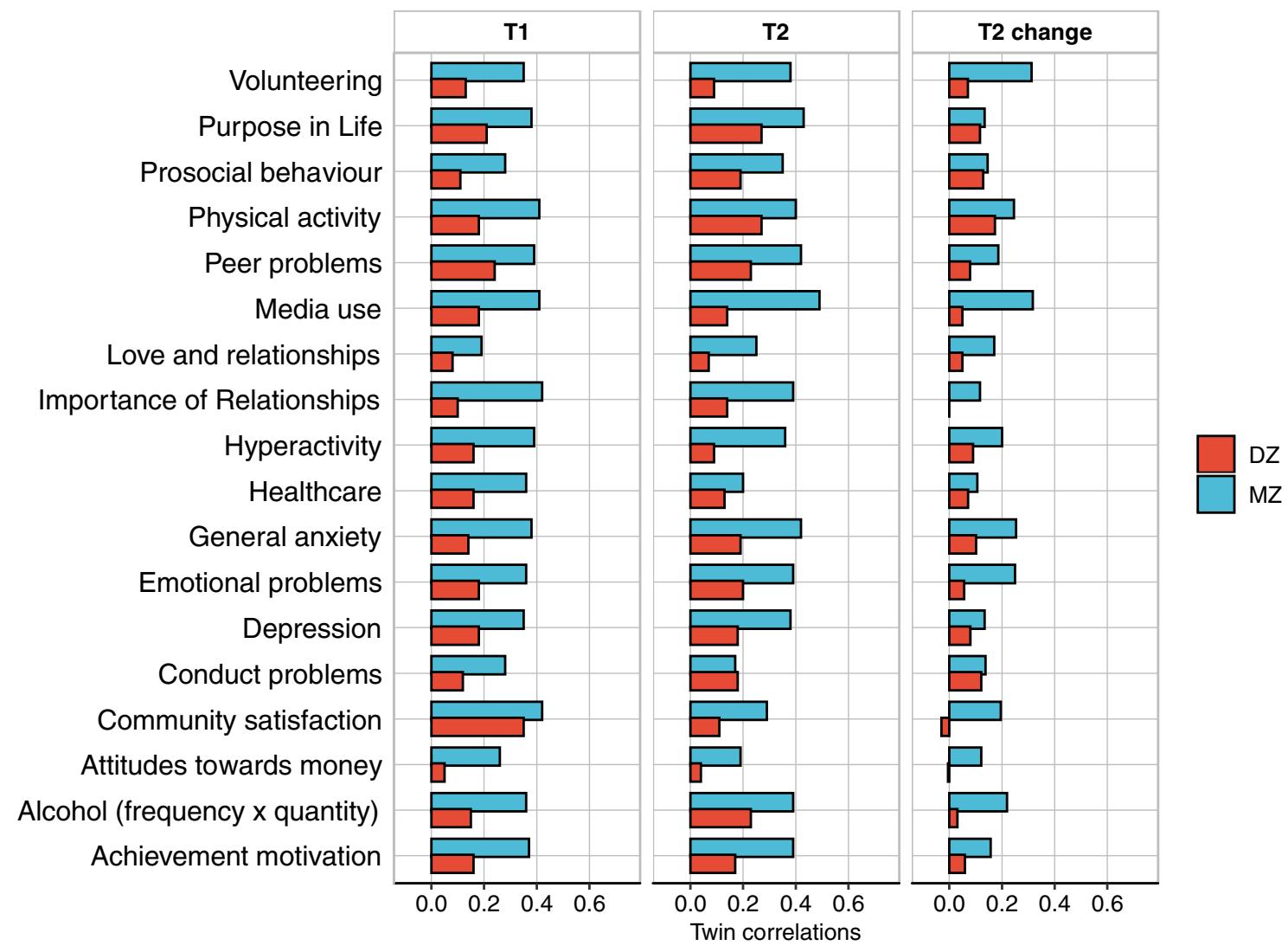

Fig. 3 Correlations between MZ and DZ twin pairs for all measures at T1, T2 and T2 change

correlations for identical and non-identical twins were similar, 0.31 and 0.16 , as was the average heritability of $30 \%$, despite the COVID-19 crisis and lockdown.

Twin resemblance not explained by zygosity can be attributed to shared environment (C). In other words, the extent to which heritability does not account for the identical twin correlation is a rough index of $\mathrm{C}$. On average, $\mathrm{C}$ was negligible at T1 $(2 \%)$ and T2 (4\%).

The rest of the variance is attributed to a residual component of variance $(\mathrm{E})$ that includes non-shared environment plus unreliability of measurement. The average E was $63 \%$ at $\mathrm{T} 1$ and $66 \%$ at $\mathrm{T} 2$. Test-retest reliabilities suggest that non-shared environment accounted for about half of $\mathrm{E}$ at $\mathrm{T} 1$ and $\mathrm{T} 2$.

Deducting the component of variance due to unreliability indicates that about half of the reliable variance at $\mathrm{T} 1$ and $\mathrm{T} 2$ can be attributed to inherited DNA differences. In other words, of the total variance at $\mathrm{T} 1$ and $\mathrm{T} 2$, about $40 \%$ can, on average across the measures, be attributed to genetic factors, about $30 \%$ to non-shared environmental factors, and about $30 \%$ to unreliability of measurement. Shared environmental influence has negligible impact.

T2 change scores show lower heritabilities, $16 \%$ on average. Because T2 change is a residualised score independent of scores at T1, stable genetic influence from $\mathrm{T} 1$ to $\mathrm{T} 2$ is removed from $\mathrm{T} 2$ change scores. Thus, heritability of $\mathrm{T} 2$ change scores represents novel genetic influence at $\mathrm{T} 2$ that does not affect $\mathrm{T} 1$. This new genetic influence could be due to gene-environment interplay: gene-environment correlation, that is where environments young adults chose or were exposed to did not simply happen at random but were correlated with their genotypes; or gene-environment interaction, that is young adults responded differently to the environment (e.g. COVID-19 and associated lockdown) based on their genotypes; alternatively, the new genetic influence can be explained by maturation over the 2-year period. Shared environment, which includes not only shared rearing environment (the twin pairs grew up together in the same family) but also shared experiences during the COVID-19 crisis, has negligible effects on T2 change, $3 \%$ on average. Most of the variance of $\mathrm{T} 2$ change scores is due to the $\mathrm{E}$ component of variance, $81 \%$ on average. We cannot separate E of T2 change scores into non-shared environment and unreliability of measurement because test-retest reliability at T1 cannot be assumed to represent the reliability of $\mathrm{T} 2$ change scores. 


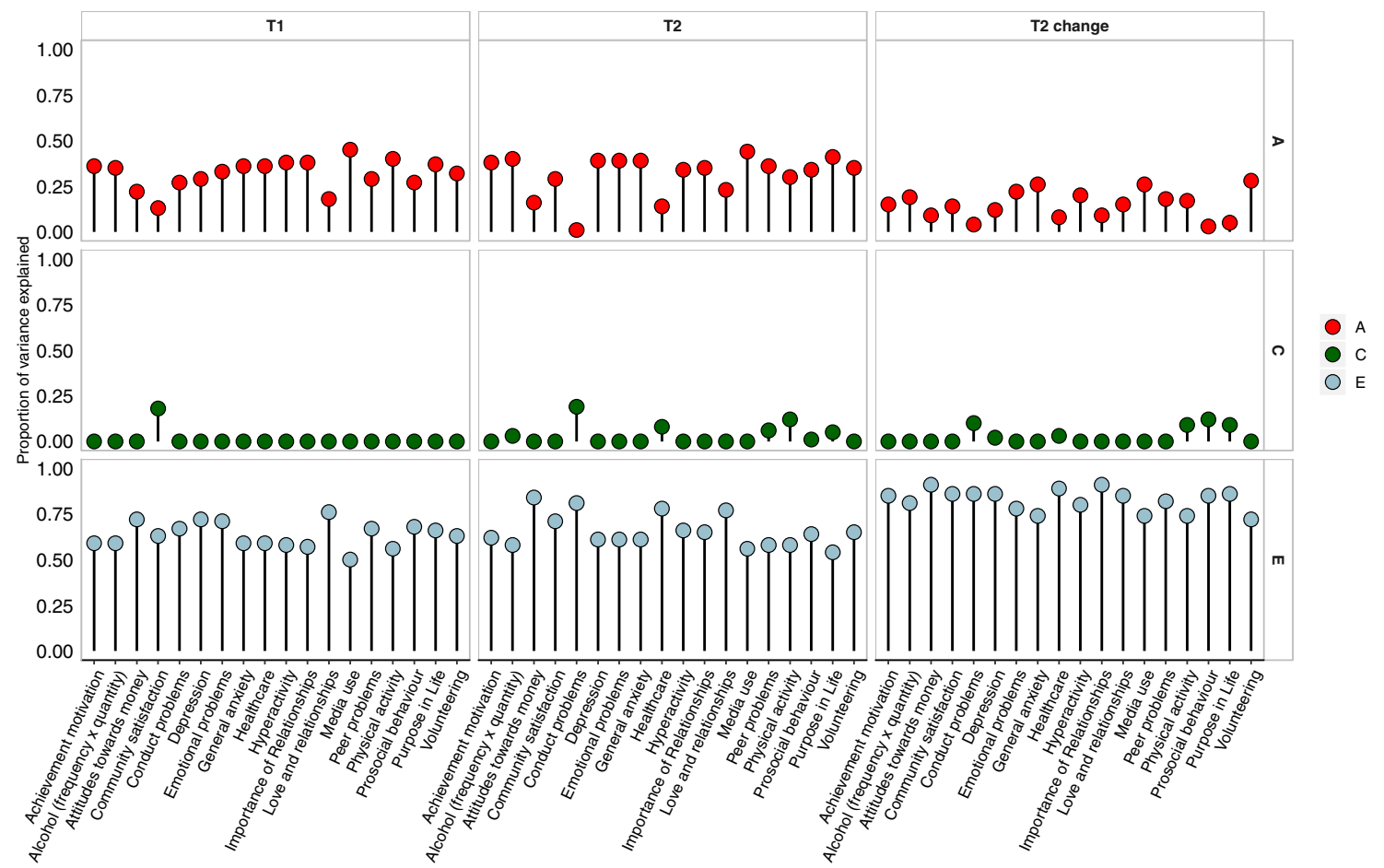

Fig. 4 Univariate model-fitting estimates

\section{Univariate model-fitting results}

These results about variance and covariance gleaned from the twin correlations are highly similar to the results of univariate model-fitting analyses of variance for T1, T2 and T2 change measures, as shown in Fig. 4. (See Supplementary Table 37 for model-fit statistics, precise ACE estimates and confidence intervals). Even though some fit statistics indicate that a better model to fit would have been AE or ADE model, and the fit statistics are only satisfactory, we decided to report full ACE model for all traits for completeness. The average broad model-fitting heritability estimates were $32 \%$ for $\mathrm{T} 1,32 \%$ for $\mathrm{T} 2$ and $15 \%$ for T 2 change, likely encompassing both additive and non-additive genetic effects. Model-fitting estimates of shared environment were $3 \%$ for T1 measures, $3 \%$ for T2 measures and $2 \%$ for T 2 change measures. Average model-fitting estimates of E were $66 \%$, $65 \%$ and $82 \%$, respectively.

\section{Bivariate model-fitting results}

The Cholesky Decomposition bivariate model-fitting model separates $\mathrm{A}, \mathrm{C}$ and $\mathrm{E}$ components of variance at T2 into variance in common with variance at $\mathrm{T} 1$ and variance at $\mathrm{T} 2$ independent of variance at T1. As explained in Methods, the model yields estimates of the extent to which the phenotypic correlation between $\mathrm{T} 1$ and $\mathrm{T} 2$ is accounted for by A, C and E. The genetic correlations are shown in the top panel of Fig. 5 (See Supplementary Fig. 2 for shared environmental and non-shared environmental correlations). The results of the Cholesky bivariate analysis are illustrated in the bottom panel of Fig. 5, with details in Supplementary Tables 38-43. Genetics accounts for $55 \%$ of the T1-T2 phenotypic correlations on average. Shared environment accounts for $4 \%$ of the phenotypic correlations on average. E influences shared at $\mathrm{T} 1$ and $\mathrm{T} 2$ are responsible for the rest of the phenotypic correlations (40\%), which could be stable non-shared environmental influences and/or correlated error.

The Cholesky model also estimates A, C and E components of variance at $\mathrm{T} 2$ independent of their respective $\mathrm{A}$, $\mathrm{C}$ and $\mathrm{E}$ components of variance at T1. These A, C and E estimates at T2 independent of those at T1 (Supplementary Tables 38-43) are, as expected, similar to the A, C and E estimates for T2 change shown in Fig. 4.

Figure 5 also shows the genetic correlations between T1 and $\mathrm{T} 2$ and shows the proportion of the phenotypic correlations (presented in Fig. 2) that can be explained by genetic, shared-environmental and non-shared environmental factors. As explained in Analyses, the Cholesky model estimates the genetic contribution to phenotypic stability from T1 to $\mathrm{T} 2$, which includes the genetic correlation. The genetic correlation is the correlation between genetic effects at $\mathrm{T} 1$ and 
Fig. 5 Bivariate model-fitting estimates. Genetic correlations are presented in the top panel. The bottom panel shows the proportion of the phenotypic correlation that is explained by $\mathrm{A}, \mathrm{C}$ and $\mathrm{E}$

\section{Volunteering \\ Purpose in life \\ Prosocial behaviour \\ Physical activity \\ Peer problems \\ Media use \\ Love and relationships \\ Importance of relationships \\ Hyperactivity \\ Healthcare \\ General anxiety \\ Emotional problems \\ Depression \\ Conduct problems \\ Community satisfaction \\ Attitudes towards money \\ Alcohol freq $x$ quant \\ Achievement motivation}

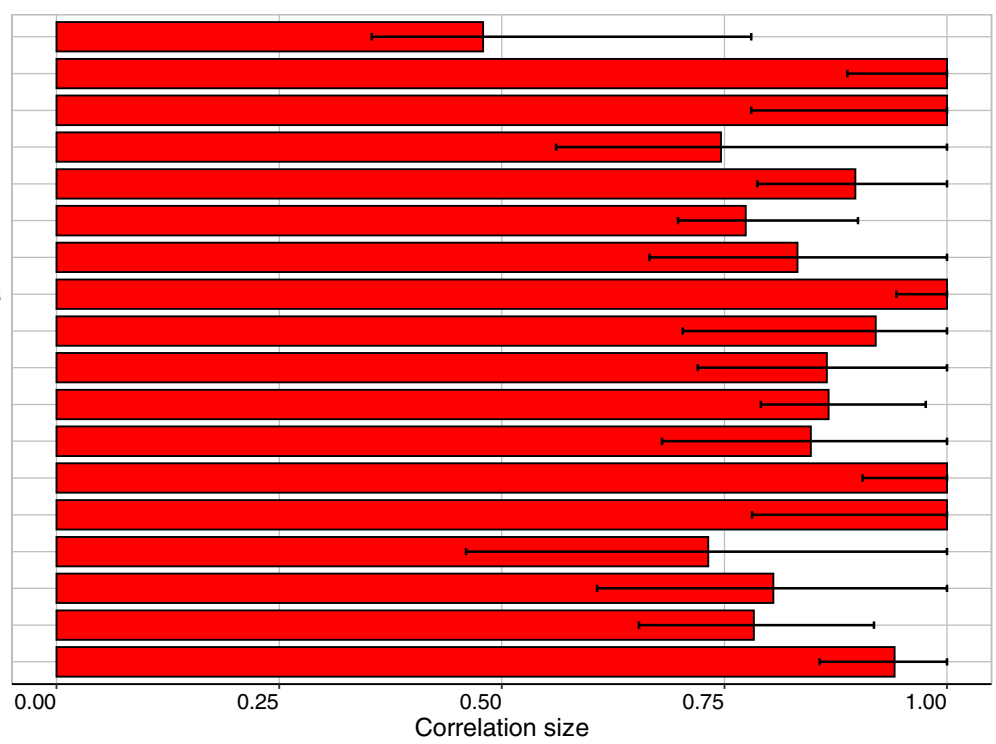

Volunteering

Purpose in life

Prosocial behaviour

Physical activity

Peer problems

Media use

Love and relationships

Importance of relationships

Hyperactivity

Healthcare

General anxiety

Emotional problems

Depression

Conduct problems

Community satisfaction

Attitudes towards money

Alcohol freq $x$ quant

Achievement motivation

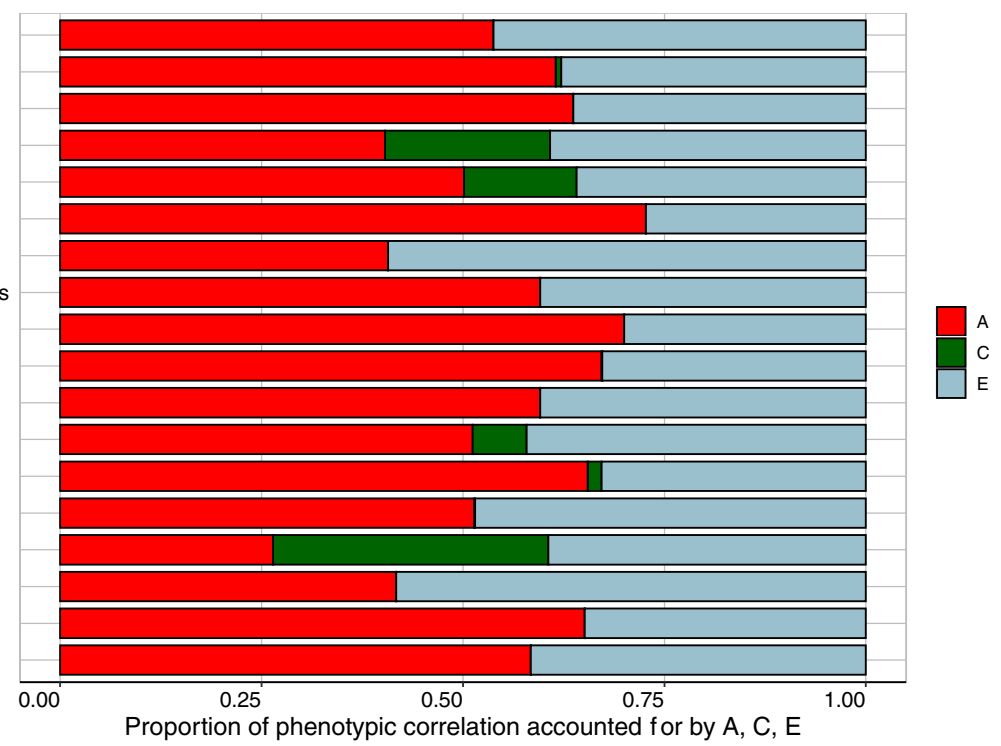

$\mathrm{T} 2$ independent of the $\mathrm{T} 1$ and $\mathrm{T} 2$ heritabilities. The genetic correlations averaged 0.91 , and most of their $95 \%$ confidence intervals included 1.0, indicating that genetic effects at $\mathrm{T} 2$ were substantially correlated with genetic effects at T1, despite the COVID-19 crisis and lockdown, although it should be noted that the heritabilities for diverse traits are modest to moderate.

\section{Twins locked down together vs apart}

Finally, we investigated possible moderators of the univariate results. The most novel moderator is whether the twins were locked down together or living apart during lockdown. Lockdown presents a quasi-experimental test of contemporary shared environments by comparing results for the $28 \%$ of twins living together during lockdown and those living apart. If shared lockdown experiences were important, twins locked down together should be more similar than twins living apart during lockdown. On the basis of the generally weak effects of shared environment, we predicted that environmental effects due to living together during lockdown are negligible.

At first this prediction seemed wrong because the average twin correlation for twin pairs locked down together (0.30) was higher than the correlation for twin pairs living apart during lockdown (0.23), although this difference was not significant $(p=0.051)$. However, this possible effect of shared environments might be a genetic effect in disguise because identical twins locked down together more often than non-identical twins ( $32 \%$ vs $25 \%$ ). Results of univariate model-fitting separately for twins locked down together 


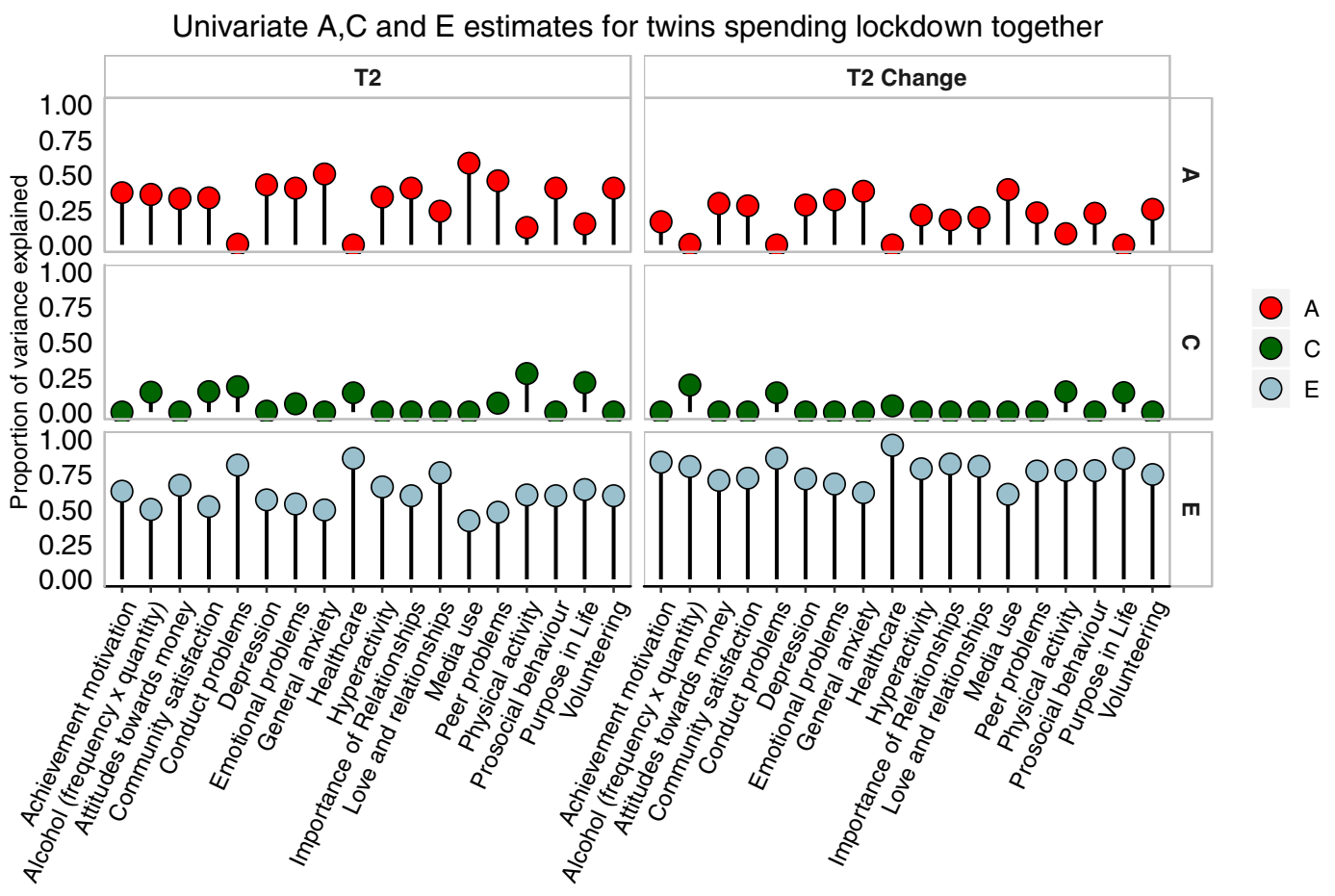

Univariate $A, C$ and $E$ estimates for twins spending lockdown apart

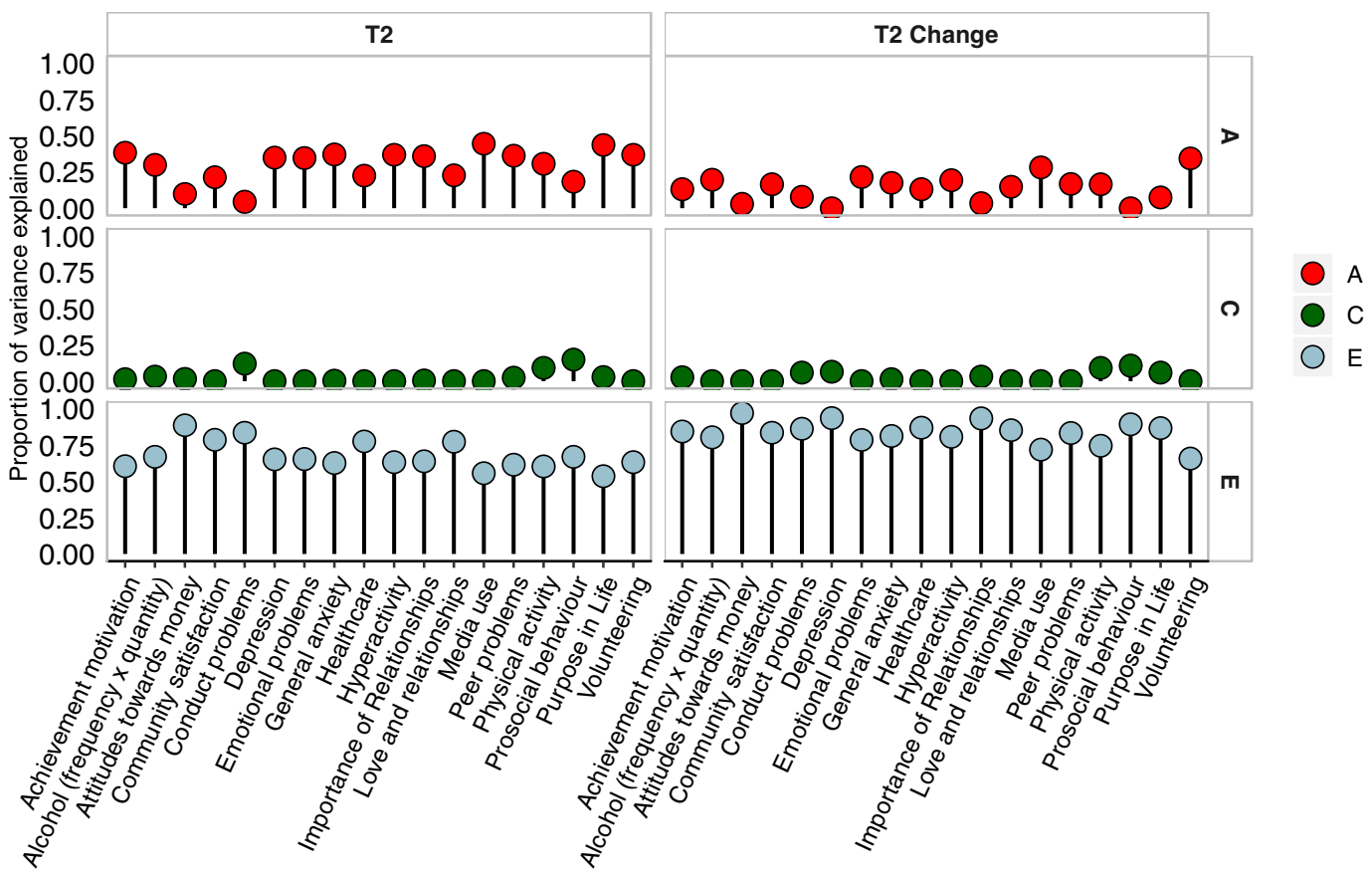

Fig. 6 Univariate model-fitting estimates for twins in lockdown together (top panel) vs. twins in lockdown apart (bottom panel)

vs apart (Fig. 6) are consistent with the notion that the apparent effect of shared environments might be mediated in part genetically (Supplementary Table 44-49 for modelfitting results including the $95 \%$ confidence intervals). For T2 scores, twins together yielded a slightly higher average estimate of shared environmental influence compared to twins apart (0.07 vs 0.03), suggesting some very slight increase in true shared environmental influence. However, twins together also yielded a slightly higher average estimate of genetic influence compared to twins apart $(0.33 \mathrm{vs}$ 0.30 ), which could be the result of genetically influenced selection for being locked down together, which would be 
an example of gene-environment correlation. However, a great deal of caution is warranted in these interpretations because the difference in phenotypic correlations for twins locked down together vs apart is not significant, and our design has negligible power to detect significant differences of this magnitude for A and C.

Power to detect significant differences for such small effects is negligible. Nonetheless, further support for the hypothesis that the apparent $\mathrm{C}$ effect of being locked down together is not really $\mathrm{C}$ comes from finding nearly identical $\mathrm{A}$ and $\mathrm{C}$ estimates pre-existing at $\mathrm{T} 1$ : $\mathrm{A}$ and $\mathrm{C}$ are 0.33 and 0.06 for twins together and 0.30 and 0.03 for twins apart. Results of $\mathrm{T} 2$ change scores provides additional confirmation in that a similar pattern emerged: $\mathrm{A}$ and $\mathrm{C}$ are 0.19 and 0.04 , respectively, for twins together and 0.14 and 0.02 for twins apart.

\section{Other moderators}

We also considered other potential moderators. For example, similar to being locked down together or apart, gender is a dichotomous variable that is the same for both members of a twin pair (when opposite-sex non-identical twins are excluded). Separate univariate analyses for male and female twins yielded similar results. These model-fitting results are presented in Supplementary Tables 50 and 51.

For the continuous moderator of family SES and for moderators that can be discordant for members of a twin pair (losing a job/financial difficulties, living conditions during lockdown, COVID-19 symptoms, impact of COVID-19 on family health and financial situation, worries of infection and impact on health, change in sleep habits), we corrected T2 and $\mathrm{T} 2$ change scores for these moderators and repeated the analyses. ACE estimates were similar when we compared estimates before and after correction for these moderators. These model-fitting results are included in Supplementary Tables 52-66.

\section{Discussion}

How much has the COVID-19 crisis changed young adults psychologically following the unprecedented COVID-19 pandemic and one month of lockdown? As expected, the 30 measures in our study yielded many statistically significant changes in means. The largest changes in the negative direction were reduced volunteering and achievement motivation and increased hyperactivity-inattention. However, there were as many changes in the positive direction, most notably, reduced verbal peer victimisation. Changes were similar in direction and magnitude for males and females, with the single exception of general anxiety, which increased more for females than males. However, most of these mean changes have modest effect sizes, with an average $d$ of 0.24 . Importantly, while the average effects on young adults after a month of lockdown were small, it is possible that the effects of the crisis will hit harder later or that longer lockdown or the economic aftermath of the crisis will have a greater effect. We hope to investigate these possibilities with followup surveys during 2020-2021.

Why do these young adults in Great Britain show modest negative effects on average after being in lockdown for one month when it is generally assumed that the psychological effects will be substantial? Part of the answer is that research often focuses on statistical significance and mean differences rather than considering effect size and individual differences. With our large sample size, nearly all variables show significant mean differences, but they accounted for less than two percent of the variance on average. Another reason might be methodological. In the present study we did not focus on participants' subjective reports of how the COVID-19 crisis changed them. Instead, at T2, we asked participants to report, for example, how depressed they felt during the month following lockdown, which we compared to their reports of depression on the same measures in 2018. We found no difference in depression on average.

Other reasons why we found few negative effects of the COVID-19 crisis could be that the lockdown was so widespread (we're all in it together spirit?) or that they are young adults (resilience? insouciance?). Concerning the insouciance hypothesis, we asked participants at $\mathrm{T} 2$ how much they were worried about their physical health and mental health during the month since lockdown. The frequency of those reporting that they were moderately, very, or extremely worried was $38 \%$ for physical health and $57 \%$ for mental health. In other words, they were, quite reasonably, worried, although on average they did not change psychologically, including their symptoms of general anxiety. This can be viewed as a hopeful message that young people on average, are resilient psychologically to an experience as seismic as COVID-19 and lockdown, although these mean differences mask individual differences to COVID-19 and lockdown. It remains to be seen if similar results emerge in other countries, at other ages and after longer exposure to the crisis and its aftermath.

The focus of our study was on individual differences rather than mean differences. How much has COVID-19 shuffled the deck of individual differences? The rank order of individual differences was largely stable from $\mathrm{T} 1$ to $\mathrm{T} 2$, with stability accounting for about $70 \%$ of the reliable variance at $\mathrm{T} 1$ and $\mathrm{T} 2$ on average across the measures. We predicted increased variance in all outcomes if the COVID-19 crisis had a major impact on individual differences, but the variance remained roughly the same. In fact, the stability of all these diverse traits was similar to what we would have predicted after a 2-year period without pandemic (Class et al. 
2019; Hannigan et al. 2017; McGue et al. 1993; Roberts and Mroczek 2008; Vecchione et al. 2020). If the COVID-19 related pandemic had a substantial effect on these diverse psychological measures, then the stability of these traits would have been lower, and we would have also observed larger differences in means and variances when comparing pre- and post-lockdown measures.

From a genetic perspective, the most interesting finding was that the average genetic correlation was 0.86 , indicating that genetic effects at $\mathrm{T} 1$ were highly correlated with genetic effects at T2, despite the intervening COVID-19 crisis and lockdown. It is also interesting that $\mathrm{T} 2$ changes, which are independent of T1, show genetic influence; however, these novel genetic influences were small and are likely to be accounted for by gene-environment interplay. For example, gene-environment interaction could occur if young adults react differently to the extreme experience of lockdown based partly on their genetics. However, our analyses of moderators did not identify gene-environment interaction, although power was limited to detect these interactions. Gene-environment correlation could occur if young adults with a greater genetic predisposition towards mental illness might be more sensitive to the experience of lockdown which in turn would result in greater psychological and attitudinal changes.

The limitations of this study include the usual limitations of twin design, which are described in detail elsewhere (Knopik et al. 2017; Rijsdijk and Sham 2002). In addition, while the TEDS study has been shown to be reasonably representative, the current sample is slightly more educated (see Methods), and it is possible that the sample is slightly less affected by the current crisis. It is also possible that individuals who were affected by COVID-19, especially those isolated or hospitalised, did not complete our survey, although the impact of affected individuals is likely to be negligible in our large sample. The proportion of affected individuals in our sample was small, only $0.1 \%$ (9 individuals) had received a positive COVID-19 test, $1.1 \%$ had medical diagnoses but had not taken the test; this is comparable to national statistics at the time (April 2020) that estimated that the proportion of COVID-19 positive individuals in the population was between 0.2 and $0.5 \%$ (Office for National Statistics 2020). However, the advantage of our study is that we have used longitudinal data and have information about diverse psychological measures prior to the current crisis and a month after the lockdown from the same individuals.

An additional consideration is about the reliability of measures, especially the change scores from T1 to T2. However, we showed that the test-retest reliabilities for these measures were around 0.71 and $68 \%$ of these reliable measures between $\mathrm{T} 1$ and $\mathrm{T} 2$ were stable, as shown by the correlations between $\mathrm{T} 1$ and $\mathrm{T} 2$; the rest of the reliable measures was accounted for by change scores. Moreover, we found that the change scores showed significant heritability, providing a further indication of reliability (all measurement error loads on the non-shared environmental component in the twin model), although we cannot be sure that this change in psychological traits happened because of the lockdown or because of various other events that happened over the 2-year period, or alternatively, because of maturation during the 2 years; we hope to address this with further follow-up studies. We also note that present analyses are based on a sample drawn from the population of England and Wales, and the results could differ in countries that implemented lockdown sooner or where lockdown was stricter.

We conclude that inherited DNA differences are the major systematic force shaping individual differences in psychological traits at T2 as well as at T1. Genetic effects account for about half of the reliable psychological differences between people at $\mathrm{T} 1$ and $\mathrm{T} 2$. The environment accounts for the rest of the variance, but it is not the systematic effect of environmental factors often assumed to be important, such as shared family environment. Environmental factors of this systematic sort had negligible effects on variance at $\mathrm{T} 1$ and $\mathrm{T} 2$ and for $\mathrm{T} 2$ change. The environmental effects that make a difference are those that are not shared by twin siblings growing up in the same family or, in our study, by twins locked down together. These idiosyncratic 'nonshared' environmental factors are likely to be unsystematic, chance experiences (Plomin 2018).

Our results confirmed seven of our eight pre-registered (https://osf.io/r58be/) hypotheses. This speaks to the replicability of findings from behavioural genetic research on which these hypotheses were based, which is noteworthy given the replication crisis in science in general and in psychology in particular (Plomin et al. 2016). The exception was the hypothesis that variance at $\mathrm{T} 2$ would be greater than at $\mathrm{T} 1$, which was a prediction not based on behavioural genetic research. The consistency of results from T1 to T2 also attests to the replicability of research in behavioural genetics.

Concluding that inherited DNA differences are the major systematic force shaping who we are psychologically does not imply that novel environmental interventions, including therapeutic interventions, cannot make a difference. It should be emphasised that heritability does not imply immutability. Heritability is a descriptive statistic limited to a particular population at a particular time with a particular mix of genetic and environmental influences. Our study can be seen as an attempt to assess whether heritability changed as a function of a tectonic shift in environment, the COVID-19 crisis. We found little evidence for such change.

Concluding that the COVID-19 crisis one month after the lockdown has not on average fundamentally changed these young people psychologically is not to dismiss the pain some of them felt before or during the crisis or will continue to 
feel after the crisis ends. Even though the crisis had little effect on means and even less effect on variances and covariances, genetically driven psychological vulnerabilities are especially important targets for preventive interventions in young adults because the twenties is a pluripotent tipping point for life-long psychological problems (Arnett 2014; Smith et al. 2011).

\section{Methods}

\section{Sample}

Our sample included young adult twins born in England and Wales between 1994 and 1996 enrolled in the Twins Early Development Study (TEDS; (Rimfeld et al. 2019). TEDS recruited over 16,000 twin pairs at birth; more than 8500 twin pairs were invited to participate in TEDS' 2018 assessment. Rich behavioural data have been collected from the twins developmentally over 14 waves of assessment in 20 years of data collection. Importantly, TEDS was a representative sample of the population in England and Wales at first contact and remains reasonably representative in terms of family socioeconomic status and ethnicity (Rimfeld et al. 2019).

We used data collected when the twins were 21 to 24 years old (completed in 2018; T1) and data collected 17 April-4 May 2020, approximately one month after the lockdown in response to the COVID-19 pandemic had started (T2). For the COVID-19 assessment, we only invited the subsample of twins for whom we had email addresses, which included many unpaired twins as well as pairs. The twins were invited by email and given a link and code to use to $\log$ on to the survey, a platform created and supported by Quodit Ltd. The survey began with an information sheet and consent mechanism. Incentives included a prize draw for iPads and shopping vouchers. Ethical approval was received from King's College London Research Ethics Committee (Reference number PNM/09/10-104). Although 4 May was the cut-off used for the present analyses, we continued to collect data, which will be used in future papers.

For our analyses for this paper, we selected twin pairs in which either one or both twins had at least some T2 data plus at least some $\mathrm{T} 1$ data. Of the total sample 5355 individuals had responded at T1, 4052 individuals had responded at $\mathrm{T} 2$, and 4000 individuals had responded at both $\mathrm{T} 1$ and $\mathrm{T} 2$. Sample sizes for each measure at $\mathrm{T} 1$ and $\mathrm{T} 2$ are reported in Supplementary Tables 1 and 2. All available data were used in the analyses. The total sample size of individuals was 5714, which includes 'unpaired' twins in which data from only one member of a twin pair was available. The total number of twin pairs in which both members of a twin pair responded at both $\mathrm{T} 1$ and $\mathrm{T} 2$ was 1133 . Of these twin pairs, 537 were pairs of identical twins, 365 were pairs of samesex non-identical twins and 231 were pairs of opposite-sex non-identical twins. In order to increase the power of our twin analyses, we combined the two groups of non-identical twins using sex-corrected data, as described later.

The sample for the current data collection at $\mathrm{T} 2$ for whom we also have data at $\mathrm{T} 1$ remains reasonably representative of the population in England and Wales for some key demographic characteristics. For example, our sample was similar to UK equivalents (Rimfeld et al. 2019) for ethnicity (94\% white vs $93 \%$ ), father employed ( $94 \%$ vs $91 \%$ ), and mother employed ( $47 \%$ vs $50 \%)$. However, the twins' parents were somewhat more educated: father with A-levels or higher (54\% vs $47 \%$ ) and mother with A-levels or higher (46\% vs $35 \%)$. The twins themselves were more likely to attend university ( $58 \%$ vs $42 \%$ ), and they were also more likely to have completed three full courses of A-levels (58\% vs $42 \%$ ). Also, more females participated (63\% vs 51\%).

\section{Measures}

The T1 assessment in TEDS, which surveyed twins when they were 21-24 years old, was completed in 2018. T1 data collection included a broad range of psychological measures such as wellbeing, thoughts and attitudes, relationships and behaviours of young adults, as well as measures of physical health (Fig. 7). These existing data provided us with a unique opportunity to examine how the COVID-19 crisis has changed the lives of young adults. The T2 data collection

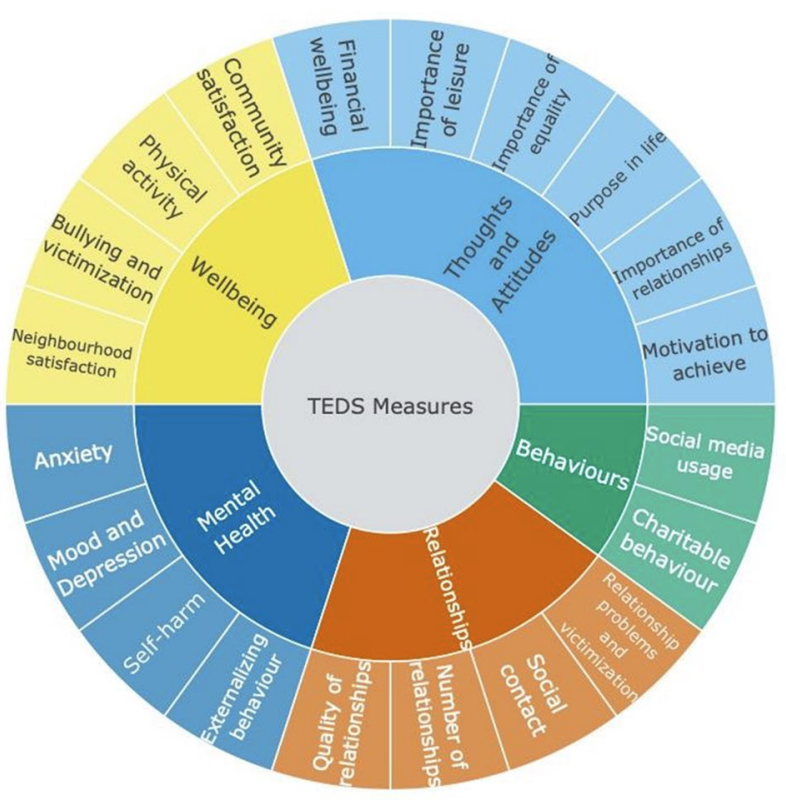

Fig. 7 Summary of measures collected at T1 and T2 


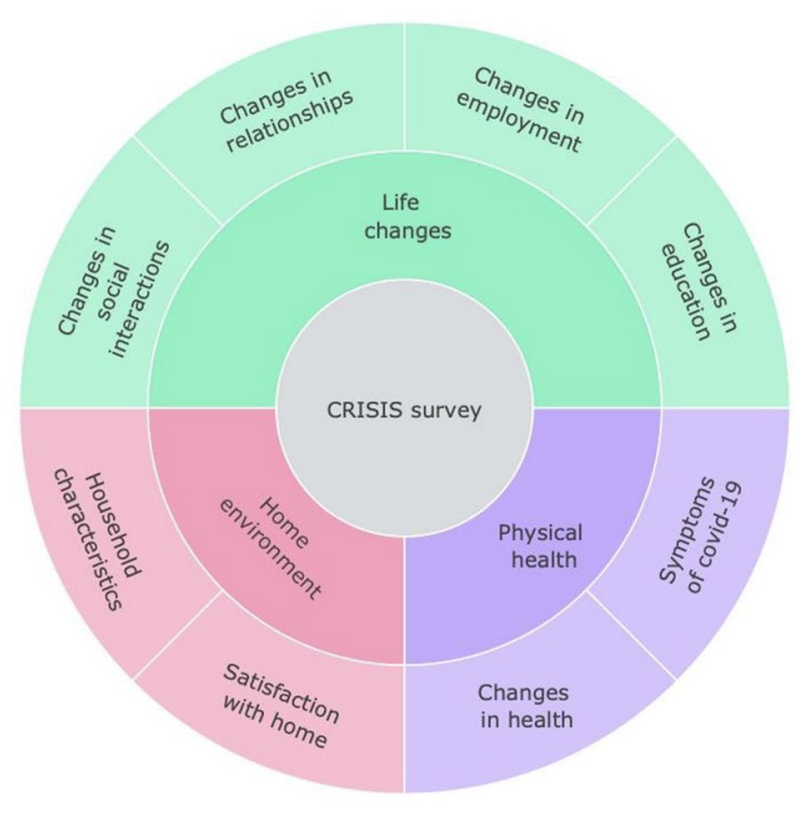

Fig. 8 Summary of CRISIS survey collected at T2

included the same measures that were collected at T1, as well as the CoRonavIruS Health Impact Survey (CRISIS; Fig. 8), developed for the purpose of assessing the physical and psychological impact of COVID-19 (Marikangas and Stringaris 2020). Data were collected using online questionnaires. The measures were administered online in an easy-touse format created by Quodit and took $15 \mathrm{~min}$ on average to complete. Participants completed the study in web browsers, on their own computers or mobile devices. Details about the measures and their references are included in Supplementary Table 11.

\section{Statistical analyses}

Our statistical analysis plan was registered in the Open Science Framework, prior to creation of the dataset and prior to analysis (https://osf.io/r58be/).

\section{Phenotypic analyses}

Means and standard deviations were calculated for all measures at $\mathrm{T} 1, \mathrm{~T} 2$ and for the change between $\mathrm{T} 1$ and $\mathrm{T} 2$ (T2 change). Change scores were calculated by correcting $\mathrm{T} 2$ scores for $\mathrm{T} 1$ scores using the regression method (by rescoring the variable as a standardised residual correcting for T1). Descriptive statistics were calculated for the entire sample, and separately for males and females. Cohen's $d$ was used to obtain an estimate of the effect sizes of the mean differences (Cohen 1988). Univariate analysis of variance (ANOVA) was used to investigate mean differences for males and females for T1, T2 and T2 change (Supplementary
Tables 1-3). Because significant, though small, sex differences emerged, explaining $0-8 \%$ of the variance in outcome measures, we corrected all scores for the mean sex differences using the regression method. Correcting for sex is important in the analysis of twin data. Members of a twin pair are identical in age and identical twins are identical for sex, and this would otherwise inflate twin estimates of shared environment (McGue and Bouchard 1984). We also corrected the measures for variation in age.

Phenotypic correlations were calculated between $\mathrm{T} 1$ and $\mathrm{T} 2$ scores for the whole sample and for males and females separately as an index of stability. We then compared the stability to test-retest reliability that was obtained in 2018 prior to T1 data collection (Supplementary Table 11). In all phenotypic analyses, we included one, randomly selected, twin from each pair to account for the non-independence of observations in the sample (i.e. twin pairs). The results remained consistent when we examined the other randomly selected half of the sample (Supplementary Tables 7-9).

Phenotypic moderation was tested using regression models. We adjusted the significance threshold for multiple testing using Bonferroni correction, therefore, all $\mathrm{p}$ values below $0.000007(0.05 / 18 * 18 * 21 ; 18$ independent variables, 18 dependent variables and 21 moderators) were considered to be significant.

\section{Genetic analyses}

Univariate twin analyses The twin design was used for univariate and bivariate genetic analyses. The twin method offers a natural experiment capitalising on the known genetic relatedness of identical (monozygotic, MZ) and non-identical (dizygotic, DZ) twin pairs. MZ twins are genetically identical and share $100 \%$ of their genes, while DZ twins share on average $50 \%$ of their segregating genes. Both MZ and DZ twins are assumed to share $100 \%$ of their shared environmental influences growing up in the same family. Non-shared environmental influences are unique to individuals, not contributing to similarity between twins. Using these known family relatedness coefficients, it is possible to estimate the relative contribution of additive genetic (A), shared environmental (C), and non-shared environmental (E) effects on the variance and covariance of the phenotypes, by comparing MZ correlations to DZ correlations. Heritability can be roughly calculated by doubling the difference between MZ and DZ correlations, $\mathrm{C}$ can be calculated by deducting heritability from MZ correlation and $\mathrm{E}$ can be estimated by deducting MZ correlation from unity (following Falconer's formula) (Rijsdijk and Sham 2002).

These parameters can be estimated more accurately using structural equation modelling, which also provides 95\% confidence intervals and estimates of model fit. The 
structural equation modelling program OpenMx was used for all model-fitting analyses (Boker et al. 2011).

Here we present twin correlations and ACE estimates for $\mathrm{T} 1, \mathrm{~T} 2$ and change scores for all variables. The difference in the intraclass correlations between MZ and DZ twin pairs can guide the decision on conducting an alternative to the ACE model, the ADE model. The ADE model partitions the variance into additive genetic (A), non-additive (or dominant) genetic (D) and non-shared environmental (E) effects. This model is fitted in cases when intraclass correlations for DZ twins are below 50\% of the MZ intraclass correlation-indicating non-additive genetic influences. Although for a few traits the DZ correlation suggested the possibility of non-additive genetic effects, we opted for running ACE models across all variables for three key reasons: first, despite our large sample size, we lacked power to detect non-additive variance reliably; second, conducting the same models across all traits allowed us to meaningfully compare the results across all measures; third, even in studies equipped with the necessary power to detect non-additive genetic effects, it is rare to find a significant contribution of $\mathrm{D}$ (and C) for self-reported psychological traits measured in adulthood (Knopik et al. 2017; Rijsdijk and Sham 2002). Therefore, the estimates derived indicate broad heritability, encompassing both additive and non-additive genetic effects.

Bivariate twin analyses These univariate analyses can be extended to bivariate analyses to investigate the aetiology of covariance between two traits. The bivariate genetic method decomposes the covariance between traits into additive genetic (A), shared environmental (C), and non-shared environmental (E) components by comparing the cross-trait cross-twin correlations between MZ and DZ twin pairs. This method also enables estimation of the genetic correlation $(r \mathrm{G})$, indicating the extent to which the same genetic variants influence two traits or measures of the same trait at two times. The shared environmental correlation $(r \mathrm{C})$ and non-shared environmental correlation $(r \mathrm{E})$ are estimated in a similar manner (Knopik et al. 2017; Rijsdijk and Sham 2002). We used bivariate genetic modelling to calculate $\mathrm{rG}$, $\mathrm{rC}$ and $\mathrm{rE}$ between $\mathrm{T} 1$ and $\mathrm{T} 2$ measures.

In addition, we investigated possible moderation for the aetiology of individual differences in T2 and change scores following the COVID-19 lockdown. For dichotomous moderators that are the same for both members of twin pairs (i.e., twins locked down together versus apart and gender), we calculated ACE estimates separately for each group and compared the univariate ACE estimates between groups. For continuous moderators (SES) and for moderators that were mostly discordant for members of twin pairs (e.g. COVID-19 symptoms, losing a job/financial difficulties, living conditions during lockdown), we corrected the trait scores for the moderator using the regression method and repeated the analyses. We then compared the univariate ACE estimates before and after the correction.

Bivariate Cholesky decomposition The Cholesky decomposition (see Supplementary Fig. 3) allows for examination of common and independent genetic (A), shared environmental (C) and non-shared environmental (E) effects on the covariance of two or more traits (Neale et al. 2005). The model assesses the independent contribution of a predictor variable to the variance in the outcome variable, after accounting for the variance accounted for by other predictors. As illustrated by the path diagram in Supplementary Fig. 2, the genetic and environmental variance in the outcome $(\mathrm{y})$ is calculated after accounting for the variance that is explained by the predictor previously entered in the model (x). As for hierarchical regression analysis, the order in which variables are entered in a Cholesky decomposition is of importance. Given the temporal succession between variables, and the fact that we were interested in examining the etiology of change and continuity between $\mathrm{T} 1$ and $\mathrm{T} 2$, we entered $\mathrm{T} 1$ measures first in the bivariate Cholesky decomposition, followed by $\mathrm{T} 2$ measures.

Supplementary Information The online version contains supplementary material available at https://doi.org/10.1007/s10519-021-10050-2.

Acknowledgements We gratefully acknowledge the on-going contribution of the participants in the Twins Early Development Study (TEDS) and their families. TEDS is supported by a programme Grant to R.P. from the UK Medical Research Council (Grant Nos. MR/V012878/1 and previously MR/M021475/1), with additional support from the US National Institutes of Health (Grant No. AG046938). The research leading to these results has also received funding from the European Research Council under the European Union's Seventh Framework Programme (FP7/2007-2013)/ grant agreement $n^{\circ}$ 602768. K.R. is supported by a Sir Henry Wellcome Postdoctoral Fellowship. S.v.S. is supported by a Jacobs Foundation Early Career Fellowship (2017-2019).

Author contributions $\mathrm{KR}, \mathrm{MM}, \mathrm{SvS}$, and RP conceived and designed the COVID-19 survey in TEDS, with help from J-BP, AGA and AEP, NGS and KLS constructed the online platform and supported the online survey. AS created the CRISIS measure and advised on its use. RO, $\mathrm{AM}$, and AEP contacted the twins and obtained the data, with finances handled by LW, AM constructed and curated the dataset. KR, MM, and AEP analysed the data. KR, MM, SvS and RP wrote the paper, which was revised by all authors, who approved the final draft of the paper. All authors contributed to selecting and validating measures at $\mathrm{T} 1$.

Data availability Data for this study came from the Twins Early Development Study (TEDS). Researchers can apply for access to the data: https://www.teds.ac.uk/researchers/teds-data-access-policy.

\section{Compliance with ethical standards}

Conflict of interest Kaili Rimfeld, Margherita Malancini, Andrea Allegrini, Amy E. Packer, Andrew McMillan, Rachel Ogden, Louise Webster, Nicholas G. Shakeshaft, Kerry L. Schofield, Jean-Baptiste 
Pingault, Argyris Stringaris, Sophie von Stumm, and Robert Plomin declare no competing interests. N.G.S. and K.L.S. are CEOs of Quodit, the company that constructed the online platform and supported the online survey, although this presents no competing interests.

Human and Animal Rights and Informed consent All procedures performed in studies involving human participants were in accordance with the ethical standards of the institutional and/or national research committee and with the 1964 Helsinki Declaration and its later amendments or comparable ethical standards. Ethical approval was received from King's College London Research Ethics Committee (Reference number PNM/09/10-104). Participants were provided with information about the study, including their rights as participants, and provided informed consent.

Open Access This article is licensed under a Creative Commons Attribution 4.0 International License, which permits use, sharing, adaptation, distribution and reproduction in any medium or format, as long as you give appropriate credit to the original author(s) and the source, provide a link to the Creative Commons licence, and indicate if changes were made. The images or other third party material in this article are included in the article's Creative Commons licence, unless indicated otherwise in a credit line to the material. If material is not included in the article's Creative Commons licence and your intended use is not permitted by statutory regulation or exceeds the permitted use, you will need to obtain permission directly from the copyright holder. To view a copy of this licence, visit http://creativecommons.org/licenses/by/4.0/.

\section{References}

Arnett JJ (2014) Emerging adulthood: the winding road from the late teens through the twenties, 2nd edn. Oxford University Press, Oxford

Boker S, Neale M, Maes H, Wilde M, Spiegel M, Brick T, Fox J (2011) OpenMx: an open source extended structural equation modeling framework. Psychometrika 76:306-317. https://doi.org/10.1007/ s11336-010-9200-6

Brooks SK et al (2020) The psychological impact of quarantine and how to reduce it: rapid review of the evidence. Lancet 395:P912920. https://doi.org/10.1016/S0140-6736(20)30460-8

Class QA, Van Hulle CA, Rathouz PJ, Applegate B, Zald DH, Lahey BB (2019) Socioemotional dispositions of children and adolescents predict general and specific second-order factors of psychopathology in early adulthood: a 12-year prospective study. J Abnor Psychol. https://doi.org/10.1037/abn0000433

Cohen J (1988) Statistical power analyses for the behavioral sciences, 2nd edn. Lawrence Erlbaum Associates, Hillsdale, NJ

Furr JM, Comer JS, Edmunds JM, Kendall PC (2010) Disasters and youth: a meta-analytic examination of posttraumatic stress. J Consult Clin Psychol 78:765-780. https://doi.org/10.1037/a0021482

Galea S, Merchant RM, Lurie N (2020) The mental health consequences of COVID-19 and physical distancing: the need for prevention and early intervention. JAMA Intern Med. https://doi. org/10.1001/jamainternmed.2020.1562

Hannigan LJ, Walaker N, Waszczuk MA, McAdams TA, Eley TC (2017) Aetiological influences on stability and change in emotional and behavioural problems across development: a systematic review. Psychopathology Rev. https://doi.org/10.5127/pr.038315

Hanscombe KB, Trzaskowski M, Haworth CMA, Davis OSP, Dale PS, Plomin R (2012) Socioeconomic status (SES) and children's intelligence (IQ): in a UK-representative sample SES moderates the environmental, not genetic, effect on IQ. PLoS ONE 7(2):e30320. https://doi.org/10.1371/journal.pone.0030320
Harris JR (1998) The nurture assumption: why children turn out the way they do. The Free Press, New York

Holmes EA, O'Connor RC, Perry VH, Tracey I, Wessely S, Arseneault L, Bullmore E et al (2020) Multidisciplinary research priorities for the COVID-19 pandemic: a call for action for mental health science. Lancet Psychiatry. https://doi.org/10.1016/S2215 -0366(20)30168-1

Knopik VS, Neiderhiser JM, DeFries JC, Plomin R (2017) Behavioral genetics, 7th edn. Worth, New York

McGue M, Bouchard TJ (1984) Adjustment of twin data for the effects of age and sex. Behav Genet 14:325-343. https://doi.org/10.1007/ BF01080045

McGue M, Bacon S, Lykken DT (1993) Personality stability and change in early adulthood: a behavioral genetic analysis. Dev Psychol. https://doi.org/10.1037/0012-1649.29.1.96

Merikangas K, Stringaris A (2020) The CoRonavIruS Health Impact Survey (CRISIS). Adult self-report baseline form. argyris.stringaris@nih.gov

Neale MC, Boker SM, Bergeman CS, Maes HH (2005) The utility of genetically informative data in the study of development. In Methodological Issues in Aging Research (pp. 269-327). https:// doi.org/10.4324/9781315820989-14

Neria Y, Galea S, Norris FH (2009) Disaster mental health research: current state, gaps in knowledge, and future directions. In: Neria Y, Galea S, Norris FH (eds) Mental health and disasters. Cambridge University Press, Cambridge

Office for National Statistics (2020) Coronavirus and depression in adults, Great Britain: June 2020. (June), 1-17. Retrieved from https://www.ons.gov.uk/peoplepopulationandcommunity/wellb eing/articles/coronavirusanddepressioninadultsgreatbritain/june2 020\#measuring-the-data

Plomin R (2018/2019) Blueprint: how DNA makes us who we are. London: Allen Lane/Penguin.

Plomin R, Daniels D (1987) Why are children in the same family so different from each other? (With Open Peer Commentary and Response). Behav Brain Sci 10:1-60. https://doi.org/10.1017/ S0140525X00055941

Plomin R, DeFries JC, Knopik VS, Neiderhiser JM (2016) Top 10 replicated findings from behavioral genetics. Perpect Psychol Sci 11:3-23. https://doi.org/10.1177/1745691615617439

Polderman TJC et al (2015) Meta-analysis of the heritability of human traits based on fifty years of twin studies. Nat Genet 47:702-709. https://doi.org/10.1038/ng.3285

Rijsdijk FV, Sham PC (2002) Analytic approaches to twin data using structural equation models. Brief Bioinform 3(2):119-133. https ://doi.org/10.1093/bib/3.2.119

Rimfeld K, Malanchini M, Spargo T, Spickernell G, Selzam S, McMillan A, Dale PS, Eley TC, Plomin R (2019) Twins early development study: a genetically sensitive investigation into behavioral and cognitive development from infancy to emerging adulthood. Twin Res Hum Genet 22:508-513. https://doi.org/10.1017/ thg. 2019.56

Roberts BW, Mroczek D (2008) Personality trait change in adulthood. Curr Dir Psychol Sci. https://doi.org/10.111 $1 / \mathrm{j} .1467-8721.2008 .00543 . x$

Smith C, Christoffersen K, Davidson H (2011) Lost in transition: the dark side of emerging adulthood. Oxford University Press, Oxford

Vecchione M, Schwartz SH, Davidov E, Cieciuch J, Alessandri G, Marsicano G (2020) Stability and change of basic personal values in early adolescence: a 2-year longitudinal study. J Pers. https:// doi.org/10.1111/jopy. 12502

Publisher's Note Springer Nature remains neutral with regard to jurisdictional claims in published maps and institutional affiliations. 